\title{
Historia
}

\section{El rol enfermero. Cambios más significativos entre ayudante técnico sanitario y diplomado universitario en Enfermería.}

\author{
Nurse role. Most significant changes between ATS \\ (sanitary technician) and DUE (university diploma in nursing).
}

Da enfermeira role. Alterações mais significativas entre ATS (assistên-
cia técnica paramedico) e DUE (diploma universitário em enfermagem)

\author{
Nuria Martínez Cadaya ${ }^{1}, M^{a}$ Luz Fernández Fernández ${ }^{2}$ \\ ${ }^{1}$ Diplomada en Enfermería. Servicio Cántabro de Salud. \\ ${ }^{2}$ Licenciada en Historia. Máster Interuniversitario de Historia Contemporánea. Diplomada en Enfermería. Profesora E.U.E. \\ "Casa de Salud Valdecilla" U. de Cantabria. \\ Cómo citar este artículo en edición digital: Martínez Cadaya N; Fernández Fernández, $M^{a} L$. (2012) El rol enfermero. Cambios \\ más significativos entre Ayudante Técnico Sanitario y Diplomado Universitario en Enfermería. \\ Cultura de los Cuidados. (Edición digital) 16, 33. Disponible en: <http://dx.doi.org/10.7184/cuid.2012.33.03> \\ Correspondência: Nuria Martínez Cadaya. C/ Joaquín Bustamante $N^{\circ} 5,10^{\circ} \mathrm{B}$. \\ C.P. 39011 Santander. Cantabria. Dirección de correo: nuria_mc85@hotmail.com. Telf.: 646426920. \\ Recibido 11/12/2011/ Aceptado: 15/02/2012
}

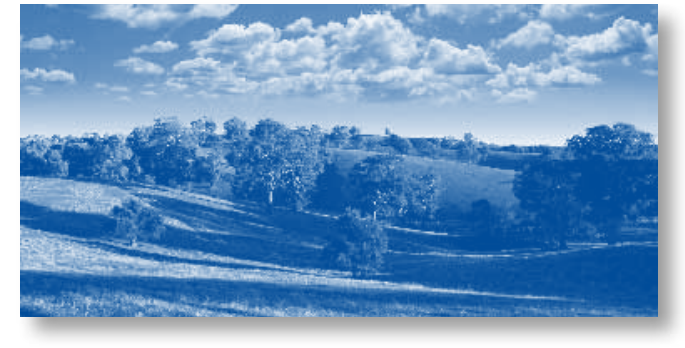

\section{ABSTRACT}

The health needs of the population have conditioned the professional role of nursing throughout history, and hence nursing functions have being redesigned, which is reflected in the professional practice. Presented below is a descriptive study carried out by a literature review that covers the period between 2000 2010 , which objective is to identify the evolution of the nurse's role in Spain in the transition from "Sanitary Technical Assistant" qualification to Nursing degree. Trough the analysis of the social, legal and formative context of the literature, it is evidenced the change in the nurse's role, changing its orientation towards the management of comprehensive care to the individual, expanding its roles to welfare, educational, management and researcher fields and using a scientific methodology based on the knowledge of their own discipline. However, these changes can be noticed more in the educational field than in the welfare field. It is concluded that the nurse's role has suffered significant changes since the implementation of the university studies although it does not achieve its real effectiveness. It is essential to deeply reflect on the reasons that prolong this situation and adopt the needed measures to fully assume the autonomous role.

Key words: Nurse's Role; Nursing Care; History of Nursing. 


\section{RESUMO}

As necessidades de saúde da população condicionaram o papel do profissional de enfermagem ao longo da historia, de modo que foram remodelando as funções de enfermaria refletindo assim na pratica profissional. A continuação apresenta-se um estudo de caráter descritivo através de uma revisão bibliográfica que abrange o período entre os anos 2000-2010 e cujo objetivo é identificar a evolução do papel do enfermeiro na Espana durante a transição de ajudante técnico-sanitario (A.T.S.) a diplomado universitário em enfermaria (D.U.E.). Através da analise do contexto social, legal e informativo da bibliografia se demonstra a mudança do papel do enfermeiro modificando sua orientação com relação à administração dos cuidados integrais do individuo, expandindo as suas funções ao nível assistencial, docente, de gestão e investigação e aplicando um método científico baseado nos conhecimentos da própria disciplina. No entanto, essas mudanças são mais visíveis no plano educacional que no plano assistencial. Concluímos que o papel do enfermeiro sofreu mudanças significativas desde o inicio dos estudos universitários, mas não chegou à verdadeira eficácia do mesmo. É imprescindível analisar profundamente sobre as razões que prolongam esta situação e tomar as medidas necessárias para assumir plenamente o papel autônomo.

Palavras-chave: Papel do Professional de Enfermagem; Cuidados de Enfermagem; História da Enfermagem.

\section{RESUMEN}

Las necesidades de salud de la población han condicionado el rol profesional de la enfermería a lo largo de la historia, de manera que se han ido remodelando las funciones enfermeras reflejándose en la práctica profesional. Se presenta a continuación un estudio de carácter descriptivo realizado mediante una revisión bibliográfica que abarca el periodo establecido entre los años 2000-2010 cuyo objetivo es identificar la evolución del rol enfermero en España en la transición de A.T.S. a D.U.E. A través del análisis del contexto social, legal y formativo de la bibliografía se evidencia el cambio en el rol enfermero modificando su orientación hacia la administración de cuidados integrales al individuo, ampliando sus funciones al ámbito asistencial, docente, de gestión e investigador y empleando una metodología científica basada en los conocimientos de la propia disciplina. Sin embargo, estas modificaciones son más visibles en el plano docente que en el asistencial. Se concluye que el rol enfermero ha sufrido cambios significativos desde la puesta en marcha de los estudios universitarios aunque no se alcanza la verdadera efectividad del mismo. Es imprescindible reflexionar en profundidad a cerca de los motivos que prolongan esta situación, y adoptar medidas necesarias para asumir plenamente el rol autónomo.

Palabras clave: Rol de la enfermera; Atención de Enfermería; Historia de la Enfermería.

\section{INTRODUCCIÓN}

La promulgación en 1857 de la Ley de Instrucción Pública supuso la primera referencia legal en la construcción la disciplina enfermera al reconocerse los estudios de Practicante y Matrona, no así los de enfermera, que deberían esperar a inicios del siglo XX concretamente por la Orden de 7 de Mayo de 1915.

Pero sin lugar a dudas, uno de los cambios trascendentales para la Enfermería españo- 
la sería la unificación de las tres titulaciones existentes, Practicante, Matrona y Enfermera, en una sola, Ayudante Técnico Sanitario en 1953, cuando tras la adhesión de España a la OMS, nuestro país debió asumir las directrices en materia sanitaria marcadas por este Organismo. Esta nueva titulación marcaría un hito para la Enfermería española ya que entre otras modificaciones, se elevaría el nivel de estudios de acceso a Bachillerato Elemental y la formación se estructuraría en tres años. Aún así, la función enfermera seguía quedando condicionada a las directrices médicas, como así lo marcaba la propia titulación al especificar la palabra "Ayudante", sin olvidar además, que la formación continuaba en manos de los facultativos de la Medicina.

El advenimiento de la democracia a mediados de los años setenta del pasado siglo, supondría una metamorfosis trascendental para la sociedad española que obviamente alcanzaría a la Enfermería. Por fin, y como ya ocurría en los países anglosajones, los estudios enfermeros se integrarían en la Universidad en 1977 bajo la titulación de Diplomado en Enfermería y los profesionales tomaban las riendas de su formación. A partir de este momento, comenzaban a establecerse las funciones independientes y los cuidados se centrarían en el ser humano como una entidad holística, abriéndose nuevas expectativas para la profesión.

En los últimos años, los distintos cambios educativos y legislativos han dado lugar a la aparición de nuevas funciones y competencias que sin duda modificarán la práctica profesional. En la actualidad, los estudios de Grado obligarán a los profesionales enfermeros a plantearse la redefinición de su rol, sin duda, una cuestión que no es sencilla de resolver.

Estas reflexiones sobre nuestras actuaciones y el resultado de las mismas, constituyeron el punto de partida para llevar a cabo un trabajo cuyo objetivo ha sido identificar el desarrollo y evolución del rol enfermero en España, más concretamente en la transición que sufrió de la titulación de Ayudante Técnico Sanitario a la integración en la Universidad para obtener la titulación de Diplomado en Enfermería.

\section{MATERIAL Y MÉTODO}

Este estudio es de carácter descriptivo y se ha llevado a cabo mediante una revisión bibliográfica que abarca el periodo establecido entre los años 2000 y 2010 utilizando para ello tanto fuentes primarias como secundarias.

La búsqueda bibliográfica se realizó utilizando las siguientes palabras clave: Rol de Enfermería; Atención de Enfermería; Historia de la Enfermería.

Para la realización de este trabajo se establecieron cinco fases:

$1^{\text {a }}$ Fase.- Búsqueda sistemática en cuatro bases de datos: Bibliografía española de Historia de la ciencia y de la técnica; CiberIndex; CUIDENplus y Dialnet; y dos catálogos bibliotecarios pertenecientes a la Biblioteca de la Universidad de Cantabria y Biblioteca Central de Cantabria.

$2^{\text {a }}$ Fase.- En esta etapa se analizaron manualmente los sumarios de los volúmenes publicados en los diez años establecidos para este estudio de seis revistas de enfermería publicadas en España con alto factor de impacto: Enfermería Clínica; Index de Enfermería; Cultura de los Cuidados; Metas de Enfermería; Revista Rol de Enfermería e Híades. Con respecto a estas dos últimas publicaciones se siguió un criterio diferente, así, en el caso de la Revista Rol de Enfermería se amplió el período de estudio, ya que no hay que olvidar que fue la primera publicación enfermera en nuestro país, por lo que se inició la revisión 
en el primer número publicado en 1978 hasta 2010. Por su parte, Híades es la única revista cuya temática se centra en la Historia de la Enfermería y por esto se realizó una revisión completa de todos los volúmenes publicados entre 1995 y 2008.

$3^{\text {a }}$ Fase.- Establecimiento de una serie de indicadores bibliométricos que permitieran realizar un posterior análisis de los datos expresados literalmente en los artículos:

- Revista en la que está publicado.

- Año de publicación.

- Nombre y apellidos de los autores.

- Número de autores por artículo.

- Titulación de los autores.

- Institución a la que pertenecen los autores.

- Zona geográfica a la que aparece vinculado el artículo.

- Existencia de palabras clave.

- Existencia de bibliografía.

$4^{\text {a }}$ Fase.- Las referencias legales que aparecen en los distintos artículos analizados se cotejaron en la base de datos de la Agencia Estatal Boletín Oficial del Estado (B.O.E.) y en su colección histórica, Gaceta de Madrid.

$5^{\text {a }}$ Fase.- La búsqueda bibliográfica se complementó con las aportaciones de fuentes primarias facilitadas por familiares y compañeros de profesión.

\section{RESULTADOS Y DISCUSIÓN}

El total de números de revistas analizados ha sido de 638 , de los cuales se han seleccionado para el estudio 82 artículos en los que se refleja una temática muy variada. Por ello, $\mathrm{y}$ dadas las peculiaridades del estudio, se ha procedido a mostrar de manera conjunta los resultados y la discusión estableciéndose en la misma dos tipos de análisis, uno bibliométrico y otro temático.

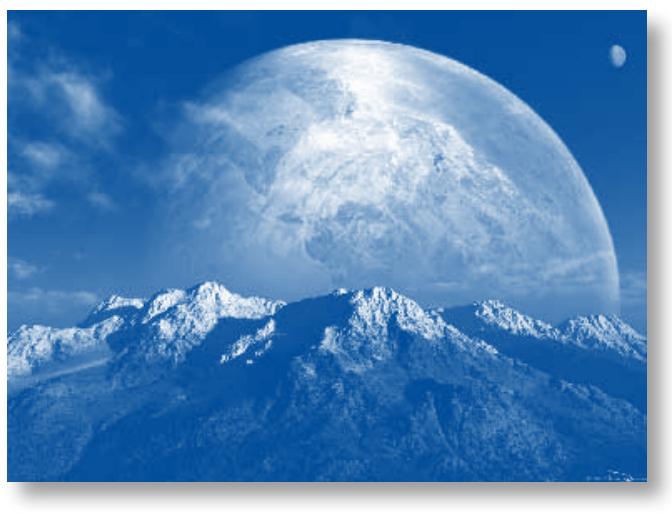

\section{ANÁLISIS BIBLIOMÉTRICO}

El primer indicador a tener en cuenta es la revista de publicación. Es necesario destacar, tal y como se muestra en la gráfica, el abundante número de artículos encontrados en la Revista Rol de Enfermería, que sobrepasa en mayoría absoluta a la suma de todos los artículos revisados de las otras cinco revistas investigadas. (Gráfico 1)

Este hecho puede explicarse dado que el periodo analizado de esta revista triplica el periodo analizado de las demás publicaciones.

El segundo dato analizado es el año de publicación del artículo. Los primeros años tras la integración en la Universidad (1978-1980) son de los de mayor productividad bibliográfica, pero en el año 2004, y sin coincidir con ninguna fecha especial, se publicó el mayor número de artículos relacionados con el tema a estudio. (Tabla 1)

Los siguientes indicadores establecidos son los autores y el número de autores por artículos. Existe una gran variabilidad de autores entre los que destaca Rosa María Alberdi Castell por ser la que más artículos ha firmado. Sin embargo, como de observa en el gráfico, el 60\% de los artículos están firmados por un solo autor. (Gráfico 2)

Para continuar con el estudio, se tuvieron en cuenta la titulación de los autores y la ins- 
titución a la que pertenecen. Las titulaciones más repetidas son enfermera y profesor de universidad. Hay que remarcar la presencia tan importante del ámbito docente en la producción de literatura científica enfermera. La universidad es la institución más destacada, pues se referencia en el $60 \%$ de los artículos; seguida de los hospitales, que se corresponden con el $10 \%$.

Otro de los indicadores analizados es la zona geográfica a la que se encuentran vinculados los autores del artículo, así se aprecia que las comunidades autónomas más productivas son Andalucía, Cataluña y Madrid.

Además, se ha valorado la existencia de palabras clave, siendo la más repetida Enfermería seguida de Cuidados, Identidad y Poder.

El último indicador bibliométrico establecido fue la existencia de bibliografía, en este sentido es interesante reseñar que solamente el $60 \%$ de los artículos contempla este apartado. Este resultado es extrapolable al resto de indicadores anteriormente expuestos en los que predomina un alto porcentaje de incumplimiento de las normas generales de publicación de las revistas.

\section{ANÁLISIS TEMÁTICO}

El 40\% de los artículos estudiados muestran la evolución formativa y legal de las distintas titulaciones de enfermería y el $60 \%$ de los artículos tiene una temática centrada en el rol enfermero pero abarcan una gran variedad de subtemas como las competencias, funciones, reconocimiento social, identidad, o perfil profesional.

En la actualidad, la profesión de enfermería va camino de completar la cuarta década dentro de la universidad, una situación, de notable mención cómo se refleja en una gran cantidad de los artículos revisados en los que

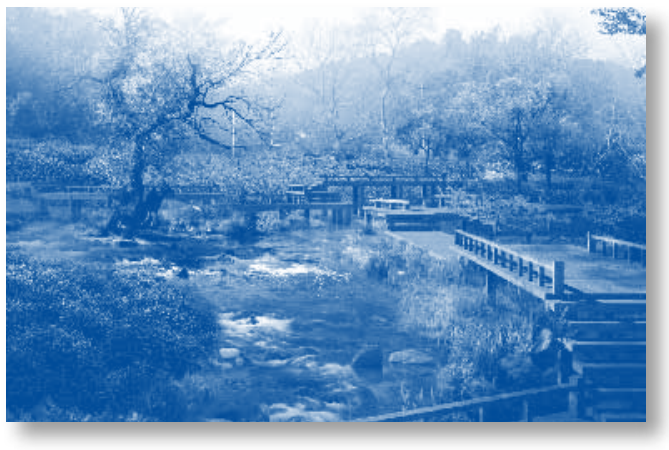

queda patente la sospecha y preocupación porque los cambios esperados no han surtido en la realidad el efecto deseado. Esta cuestión puede apreciarse en los diferentes documentos en los que se plasman algunos de los problemas que impiden según los autores, el avance profesional como es el caso de la falta de especificidad de las funciones y competencias, la ausencia de poder efectivo o la identidad no consensuada, además del reconocimiento infravalorado y la inadecuada imagen social. Es interesante reseñar que estos factores aparecen de manera recurrente indistintamente del año de publicación del artículo, ya sean de finales de los años setenta o hayan sido publicados en la actualidad. Aun así, pocos son los autores que se implican en proponer una solución a la controversia planteada, y si lo hacen, se trata de vagas indicaciones sobre el objetivo que se quiere conseguir pero sin explicar los pasos precisos para alcanzarlo.

En relación a la función asistencial que ejercen las enfermeras, son varias las teorías que sostienen que no se ha desligado del modelo biomédico centrado en la enfermedad, cuestión que reflejan varios autores como Miró (2010) quién señala que "el rol enfermero está conformado por dos dimensiones profesionales, la cuidadora o independiente y la curativa o interdependiente" y otros autores indican que "ambas son complementarias: No sería posible curar sin cuidar" (García, Sainz y Botella, 2004). 
Con respecto a lo anteriormente expuesto, debe tenerse en cuenta que en la etapa de Diplomado, al adquirir una visión integral de la persona, se pretendía enfatizar el rol independiente, pero se observa, que esta modificación no es asumida por todo el colectivo ya que como explican García y Buendía (2001) "los profesionales enfermeros se consideran en una gran mayoría, la máxima autoridad y responsables de los cuidados básicos del paciente, pero todavía no asumen de forma clara los cuidados relacionados con las necesidades básicas como la esencia de la profesión, incorporando a los cuidados de tratamiento como una parte de ella". Además, apuntan que "los enfermeros siguen organizando el trabajo por tareas y, en su mayoría, no utilizan el diagnostico enfermero ni trabajan con sus pacientes en base a un plan de cuidados". Es cierto, que esta situación podría verse condicionada por ciertas condiciones laborales como la presión asistencial, "hábitos muy arraigados, inadecuados registros" (García et al., 2001) o como explica Palacios (2007) por la propia "organización interna de los hospitales que se realiza en base a unidades médicas orientadas a la patología o a la realización de técnicas diagnosticas y terapéuticas especificas".

La misión de la enfermería es atender, cuidar, y es lo que nos identifica como profesión, de manera que algunos autores auguran que si no se desempeña esta función, y continúan destacándose "las funciones más técnicas, podría llegar a sustituirse las enfermeras por otros profesionales de categoría inferior y formación profesional técnica" (Chocarro, Guerrero, Venturini y Salvadores, 2004). Estos mismos autores, indican que la actividad asistencial recibe mayor consideración respecto al resto de funciones enfermeras. Esto es ratificado por el director del foro español de pacientes (Blancafort, 2006), el cual revela que "una de las principales demandas de las asociaciones de pacientes es la mejora y entrenamiento en habilidades de comunicación entre el colectivo de profesionales sanitarios".

La función docente, es una competencia adquirida con la titulación de Diplomado en Enfermería en la que se distinguen dos campos de actuación: la formación del personal enfermero y la información y educación sanitaria a los individuos y su comunidad. En varios documentos se plasma la incongruencia entre el perfil transmitido a los alumnos en las Escuelas, publicaciones y reuniones científicas de Enfermería y el perfil desarrollado en los centros asistenciales. Así, García et al. (2001) exponen que "los estudiantes al finalizar su formación e incorporarse al mundo laboral, les resulta difícil poner en práctica los modelos de enfermería y la metodología de trabajo aprendida, adaptándose a un sistema de funcionamiento asistencial".

La Orden de 28 de febrero de 1985 establecería los órganos de dirección de los hospitales surgiendo por primera vez las Direcciones de Enfermería y los nuevos modelos de gestión, cuestión que sin duda supondría un paso hacia delante en el reconocimiento de la profesión, estableciéndose la función gestora. Así, si tras la incorporación a la Universidad se había conseguido el reconocimiento de la autonomía de la profesión, pasándose de realizar tareas subordinadas a trabajar en equipos interdisciplinarios y multidisciplinarios, a partir de ahora, todo lo relativo a los recursos humanos y materiales enfermeros, sería responsabilidad de la gestión de la profesión.

$\mathrm{Al}$ analizar el papel de los gestores enfermeros en la consolidación del rol profesional, puede apreciarse que los resultados sobre esta función son muy variados identificándose una falta de unificación, aunque coinciden en se- 
ñalar que la Enfermería ha alcanzado puestos de reconocido poder, desempeñando cargos en el Ministerio de Sanidad como Asesoras o en las Direcciones de Gerencias Hospitalarias y de Atención Primaria. (Martínez, 1986; Durán, 2002). Por el contrario, para otros, no se ha asentado completamente esta función ya que consideran ínfima la representación de la profesión en puestos de poder (Alberdi, 1983), apuntando como posibles causas la "acomodación al sueldo y al puesto" (Montes, 2002), aunque algunos autores opinan que la realidad es que algunos profesionales no quieren aceptar cargos de poder para evitar tener que asumir responsabilidades (Lunardi, Peter y Gastaldo, 2006).

Igualmente, otro de los aspectos que se refleja en este estudio es el empeño por conseguir la formación de Grado superior y el desarrollo de las Especialidades "para facilitar el acceso real a los puestos de verdadero poder ya que la categoría profesional marca hasta dónde se puede acceder en la organización" (Cano-Caballero, 2004). Los autores señalan que a pesar de la perseverancia, no se han conseguido los logros esperados, por un lado, han pasado casi treinta años desde la inclusión de Enfermería en la Universidad hasta la aprobación del Decreto de Especialidades en el 2005, y desde entonces hasta ahora no están vigentes en su totalidad. Asimismo señalan, que la educación superior universitaria se ha alcanzado gracias a la inclusión en el Espacio Europeo de Educación Superior que clasifica la enseñanza superior en tres niveles y permite a la Enfermería por primera vez realizar cursos de Master oficial y trabajos de doctorado en la propia disciplina.

Por último, y en lo que concierne a función investigadora puede decirse que comienza a tenerse interés por "investigar en cuidados para demostrar la validez de las intervenciones enfermeras" (Alberdi, 2006), y para establecer guías y protocolos de práctica clínica con el fin de concretar criterios de actuación basados en la evidencia científica, además de la necesidad de transmitir las inquietudes y resultados obtenidos. A partir de la década de los ochenta, comienzan a surgir en España las primeras revistas especializadas en enfermería, y su incremento en número ha sido tan importante, que ha obligado a crear bases de datos bibliográficas para su gestión. Por otra parte, hay que destacar los numerosos Congresos tanto a nivel nacional como internacional y la publicación de libros y tesis doctorales. Aún así, la investigación en enfermería sigue siendo un tema muy discutido, algunos autores identifican cierta inapetencia por ella, "la actividad investigadora no es todavía asumida como un elemento más del desarrollo profesional por muchos de los enfermeros" (García et al., 2001), mientras que otros afirman que "el desarrollo de la investigación es un elemento clave para el impulso y consolidación de la profesión" (Chocarro et al., 2004; Teixidor, 2006).

\section{CONCLUSIONES}

Es evidente que se han producido cambios profundos en el rol enfermero y que se plasman:

- En la forma de pensar: Deja de centrarse en la curación de la enfermedad para cuidar la salud de los individuos y su entorno.

- En la forma de actuar: No se limita a ejercer su función asistencial y amplia sus funciones a otros ámbitos de actuación, sino que las desarrolla con autonomía y responsabilidad propia desprendiéndose del carácter subordinado y dependiente.

- En la forma de hablar: Normaliza los diagnósticos enfermeros y el empleo de una metodología científica mediante la aplicación de los Planes de Cuidados. 
A pesar de todo, estos cambios en el rol enfermero no han alcanzado los niveles deseados, y es una cuestión que se repite a lo largo de los artículos desde finales de los años 70 hasta la actualidad. Es imprescindible reflexionar en profundidad a cerca de los motivos que prolongan esta situación y adoptar las medidas necesarias para que se produzca el desarrollo efectivo del rol profesional.

Con el nuevo cambio de titulación que se esta produciendo en la actualidad, ¿solucionarán los estudios de Grado esta situación?, la respuesta nos la dará el tiempo ya que como dijo Florence Nightingale "lo importante no es lo que nos hace el destino, sino lo que nosotros hacemos con él".

\section{BIBLIOGRAFÍA}

- Alberdi, R.M. (1983). La enfermería ¿profesión femenina? Revista Rol de Enfermería, 57, 21-27.

- Alberdi, R.M. (2006). Los estudios universitarios de enfermería y sus implicaciones en la investigación en cuidados. Enfermería Clínica, 16(6), 332-335.

- Blancafort, S. (2006). ¿Qué esperan los pacientes de los profesionales sanitarios? [editorial]. Enfermería clínica, $16(1), 1-2$.

- Cano-Caballero, M.D. (2004). Enfermería y género, tiempo de reflexión para el cambio. Index de Enfermería, 13(46), 34-39.

- Chocarro, L., Guerrero, R., Venturini, C., Salvadores, P. (2004). Análisis de la identidad profesional a través de la competencia educadora de la enfermería. Cultura de los cuidados, 15, 55-62.

- Durán, M. (2002). Un homenaje a los 25 años de Enfermería en la Universidad. Revista Rol de Enfermería, 25(1), 22-30.

- García, I., Buendía, A. (2001). Identidad e identificación de la enfermería. Revista Rol de Enfermería, 24(7-8), 539-545.

- García, A. M., Sainz, A., Botella, M. (2004). La enfermería vista desde el género. Index de Enfermería, 13(46), 45-48.

- Lunardi, V., Peter, E., Gastaldo, D. (2006). ¿Es ética la sumisión de las enfermeras? Una reflexión acerca de la anorexia de poder. Enfermería Clínica, 16(5), 268-274.

- Martínez, B. (1986). Papel de la Enfermería en la Reforma Hospitalaria. Revista Rol de Enfermería, 91, 40-43.

- Miró, M. (2010). Los modelos conceptuales, una estrategia de poder con implicaciones profesionales. Enfermería Clínica, 20(6), 360-365.

- Martínez, N. (2011). El rol enfermero: Cambios más significativos entre Ayudante Técnico Sanitario y Diplomado Universitario en Enfermería. Trabajo Fin de Grado Universidad de Cantabria. [No publicado].

- Montes, J.F. (2002). La enfermería, una breve aproximación sociológica: desde, donde y hacia donde. Cultura de los Cuidados, 11, 30-39.

- Palacios, D. (2007). La construcción moderna de la enfermería. Cultura de los Cuidados, 22, 26-32.

- Teixidor, M. (2006). La Europa de la salud: Un nuevo espacio para las enfermeras. Metas de Enfermería, 9(6), 61-66.

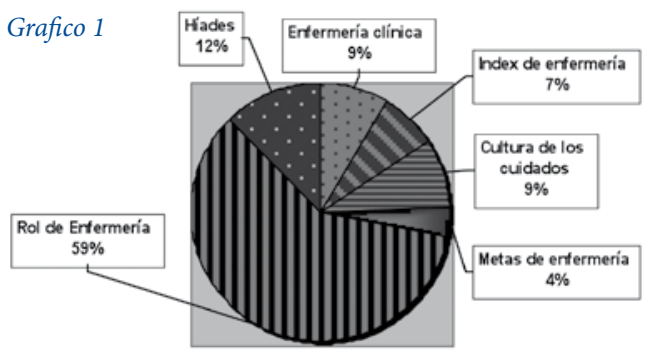

Gráfico 2

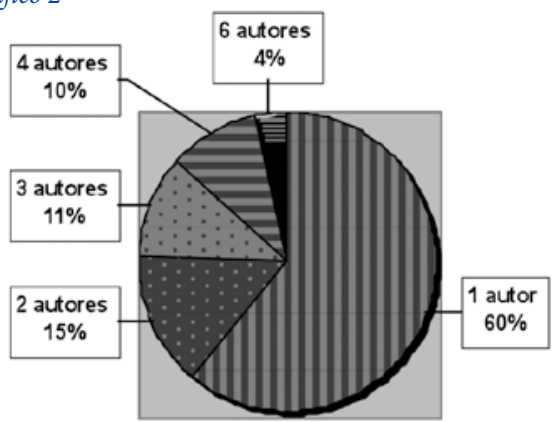

Tabla 1

\begin{tabular}{|c|c|c|c|c|c|}
\hline Año & $\mathbf{N}^{\circ}$ artículos & Año & $\mathbf{N}^{\circ}$ artículos & Año & $\mathbf{N}^{\circ}$ artículos \\
\hline 2004 & 9 & 1986 & 6 & 1978 & 4 \\
\hline 2008 & 8 & 1979 & 5 & 2002 & 4 \\
\hline 1980 & 6 & 2006 & 5 & 2009 & 4 \\
\hline
\end{tabular}

